some increase in secretion, nothing was detected. The left oronchus was similarly explored with a like result. The child was kept under observation for some time, but there was no was kept under observation of the attacks.

It has been pointed out by Killian that bronchoscopy may be of service in localizing pulmonary cavities-a matter of great importance in the surgery of the lung. From the point of view of diagnosis the following case is of some interest:

A girl, aged 8, was admitted to the medical wards suffering 'from purulent expectoration of six months' standing. The pus was ejected in considerable amount, generally once a day in the morning, and had the appearance of coming from a lung cavity. The physical signs were indefinite, being chiefly loud rhonchi over both bases. With a view to ascertain the source - of the pus a bronchoscopic examination was made, the child theing under chloroform. The right bronchus was explored theing under chloroform. The right bronchus was explored into the base of the lung and the openings of the branches ever, only a small quantity of stringy mucus. On inserting the tube in to the left bronchus a quantity of pus was ejected through it with some force. After careful mopping, most of the pus appeared to come from a branch of the bronchus serving the middle of the lung. Ten days later another examination was made the tube on this occasion being passed examination was mecter at once into the same result, a large quantity of pus coming out of the same bronchia and was about on the second day. As the general condition was improving under medical treatment this was continued.

In such a case it would be possible to pass a probe into the branch and have a skiagraph of it taken in situ, so as to facilitate localization; or bismuth might be insufflated into the cavity and a skiagraph made for the same purpose. In the event of surgical procedures, for example, pneumotomy, the presence of the probe would enable the surgeon to locate the cavity, often a matter of great difficulty in a partially-collapsed lung.

From the evidence that has been produced it appears to me abundantly clear that direct examination of the air passages is a method of the greatest utility. It is especially welcome in the treatment of that group of cases which has always been so difficult and has yielded not a few tragedies, namely, foreign bodies in the air passages, and it is earnestly to be hoped that the time has now come when workers in this country will recognize its enormous advantages.

REFERENCES.

1 Lancet, 1868, i, p. 470. 2 Diseases of Throat and Nose, vol. ii, p. 15

\section{HUMAN AND BOVINE TUBERCULOSIS.} THE DANGER OF INFECTED MILK.

By NATHAN RAW, M.D., M.R.C.P.LoND., F.R.S.EDIN., PHYSICIAN, MILL ROAD INFIRMARY, LIVERPOOL; BRITISH MEMBER OF THE INTERNATIONAL COMMITTEE FOR THE PREVENTION OF

In the British Medical Journal of 1903, 1904, and 1905, I have had the pleasure of expressing my views as to the part played by tuberculous cattle in the spread of tuberculosis amongst humans, more especially in children. The intricate and difficult problem as to the exact relationship between human and bovine tuberculosis is still unsolved, because the actual test-namely, the experimental test of inoculating humans is inapplicable, and always will be. Hence we must work by roundabout methods, making clinical observation our basis, and relying on inferences from analogy, pathological research, and minute and careful comparisons between the bacilli of various origins for our ultimate conclusions.

I would like to follow the lines of my original paper of 1903 , since I have no reason to modify the view then set forth-namely, that human and bovine bacilli are divisible into two distinct types of a common species:

\section{Typus humanus.}

Long-continued residence in a particular host has resulted in the bacilli assuming characteristics which serve to distinguish it from others, and the fact that tubercle bacilli have lived for thousands of years in human lungs would be ample evidence for assuming a difference between bacilli which had lived for centuries in cattle.

To what extent infectious agents are restricted in their activities is shown by the fact that only one of the infectious diseases in man-the bubonic plague-is shared by one of the lower animals - the rat. The plague is known to spread freely from rat to rat, from rat to man and less freely from man to man, so that some are inclined to the view that plague is primarily a rat disease.

There are other infections which may be transmitted from animals to man, but only under exceptional circumstances (Smith).

Another fact of the most profound importance in studying this problem is that for centuries man has been accustomed to feed upon cattle and their products (milk, butter, cheese, etc.), and in this way the human body has become tolerant to bovine tubercle bacilli.

Whilst I firmly believe that human and bovine bacilli are different types of parasites, yet $I$ am convinced that bovine bacilli are freely communicable to humans and are the cause of a large amount of tuberculosis in children.

Koch holds that human and bovine tuberculosis are separate and distinct diseases, and that bovine tuberculosis if conveyed to man cannot set up generalized human tuberculosis.

Koch, in addition, believes that phthisis is conveyed from one person to another by direct infection, especially from contact with an advanced case of the disease, and that this is by far the commonest cause of the spread of the disease. Von Behring, on the other hand, holds diametrically opposite views. He says that human and bovine tuberculosis are the same disease, and that nearly all tuberculosis is the result of infection during infancy by means of infected milk, and that direct infection from person to person is not proved. I venture to submit that the truth will be found between these opinions, and, whilst I firmly believe that human and bovine bacilli are distinct varieties of an original species, yet they are sufficiently distinct to produce different and characteristic lesions in the human body; and I am also convinced that bovine bacilli give rise to a large amount of tubercle in the child through infected milk.

During the last three years I have made a very large number of pure cultures of tubercle bacilli from various sources-human, bovine, and avian. My original cultures were obtained from Berlin, Copenhagen, and Lille through the kindness of Professors Koch, Bang, and Calmette. Three cultures were obtained from each laboratory, and, after growing a very large number, one is bound to admit distinctive cultural differences on the same medium between human and bovine bacilli. All are agreed on the distinctive characteristics of avian bacilli.

So much, then, for the bacteriological side, "which has received important support from the Imperial German Commission appointed to investigate the question of the relationship between human and bovine tuberculosis. They have reported entirely in favour of their being distinct varieties, and out of 23 cases of tuberculosis from human sources examined they found 6 to be of bovine origin, and all from children.

From the clinical and pathological side, my contention is considerably strengthened, and for this purpose I have examined over 4,000 cases of tuberculosis which have been under my care during the last nine years, a large number of which were children. In addition I have made over 1,600 post-mortem examinations on tuberculous cases, and most of them have been examined minutely.

Leaving for a moment the difficult question of modes of infection, I have come to the conclusion that man is attacked by two distinct varieties of tubercle, one conveyed by infection from person to person, the other received into the body through tuberculous milk and dairy produce. After examining some thousands of cases of phthisis one cannot but be impressed with the fact that the tuberculous process is confined strictly to the lungs and intestines in a very large percentage of cases-in fact, it is rare to see enlarged lymphatic glands, tuberculous joints, lupus, or peritonitis associated with true phthisis pulmonalis.

As a primary disease, phthisis seems to originate in the lungs as a result of inhalation of infected tuberculous matter, and secondarily to cause tuberculous ulceration of the intestine by swallowed sputum.

Other forms of tuberculosis, such as enlarged lymphatic glands in the neck, tuberculous peritonitis, tuberculous joints, and probably tuberculous meningitis and lupus, are most likely caused by bovine bacilli being absorbed through the digestive tract in milk and other food, such as butter, buttermilk, cheese, sausage, etc. Enlarged glands in the 
neck are, in my judgement, a grave danger. In a great many instances the infection through the fauces and tonsils by infected milk is limited by the glands which either suppurate or are removed by the surgeon. If the infection is allowed to spread downwards, the apex of the lung may be directly attacked and an extensive bovine infection of the lungs may result. Although this is rare, I have seen several instances in which I have watched the process extend from the glands to the lung. For this reason, it is of the highest importance that all tuberculous glands should be excised as quickly as they appear.

From my own observation I would say that human tubercle bacilli do not attack the ordinary lymphatic glands of the body.

I have just completed a series of 100 carefully-conducted autopsies on children dying of tuberculosis, many of them ordinary phthisis, and several of them abdominal and meningeal tuberculosis. Of this number, 38 showed tuberculous intestinal ulcers with caseating mesenteric glands, and 23 showed caseating mesenteric glands without intestinal ulcers.

From the nature of the pulmonary lesions, I am inclined to think that the tuberculous ulcers were caused by human bacilli, and that in those cases where the mesenteric glands were infected without ulceration of the intestine, the bacilli were of bovine origin.

In short, human bacilli, whether primarily swallowed or as a secondary infection in phthisis, will cause intestinal ulceration, whilst bovine bacilli ingested with milk, etc., will readily pass through the intestine, leaving no trace behind, attack the mesenteric glands, and thence spread to the lungs and all over the body.

It is possible that this may be a means in future of distinguishing the origin of an infection.

I am at present engaged in collecting information from all parts of the world as to the distribution of the various forms of tubercle and its association with tuberculosis in cattle.

Some results already received are most interesting, and tend generally to show that in those countries where tuberculous cattle are absent, or where milk is not drunk by children, the surgical forms of tuberculosis are absent, whilst phthisis pulmonalis is rife. One letter of great interest is from Siam, and is from the Government medical officer, of which the following is an extract:

As our experience in Siam would seem to support your contention that tabes mesenterica and other tuberculous affections of the serous membranes in children are probably bovine tuberculoses conveyed by milk and are not true human tuberculoses, I send you the following notes, hoping they may be of interest to you :

During eight years' practice in Bangkok I do not remember having even seen or ever having heard of any case of strumous joints, lupus, or tabes mesenterica, or, in fact, of tuberculous
affections of such nature in children. I have often spoken of this to my colleagues, but could formulate no reason until I read your paper in the BRITISH MEDICAL JOURNAL. The Siamese practically never drink cow's milk, either children or adults. Ordinary pulmonary tuberculosis, however, in adolescents or their elders is very rife in Bangkok. The same seems to hold good for China.

I have received similar confirmation from Jersey, Guernsey, Egypt, Malay, India, and Persia. When the inquiry is complete I will publish it.

A very valuable contribution to this question has just been made by Dr. Mayo, of Rochester, Minn. He says, speaking of abdominal tuberculosis:

Practising as my brother and I do in an agricultural com ${ }^{-}$ munity, we have long noticed - the relative infrequency of pulmonary tuberculosis, while abdominal, bone, joint and cannot help believing that much of the extra-pulmonary tuberculosis had to do with uncooked milk, which is a common article of diet in this country.

This testimony is of great importance, and I feel sure that until tuberculosis is reduced in dairy cattle, where it at present prevails to the extent of between 20 and 25 per cent., there will not be much reduction in the mortality from tuberculosis in children.

The suppression of tuberculosis must, in my judgement, be accomplished by attacking two sides of the questionfirst, phthisis pulmonalis; secondly, bovine tuberculosis. Phthisis pulmonalis is essentially a "house" disease, and is most rife amongst the poor and where people live in insanitary and crowded rooms, by which infection is easily conveyed to different members of a family.

Owing to improvements in hygiene and the sanitary conditions in which the poor live, it is gratifying to find that the death-rate from phthisis is steadily diminishing, but the same cannot be said of tuberculosis in children and I feel convinced that until milk free from tubercle bacilli can be supplied to children, we must not expect a marked diminution in the surgical forms of tuberculosis.

In my original paper I expressed the opinion that these two varieties of tubercle were antagonistic to each other in the human body, and that children who have suffered from bovine tuberculosis in the form of strumous glands, spinal caries, strumous joints, etc., did not develop true phthisis in later life. In fact, I argued that this mild infection conferred a certain immunity against phthisis in the same way as vaccination and small-pox.

This theory has also received great support by the work of von Behring and Römer. The only strain of tubercle bacilli which they find produces complete immunity is the human strain, and by using human bacilli they have been able to immunize thousands of cattle against bovine tuberculosis, not one of which has contracted tubercle, although repeatedly exposed to infection by living in the same stalls with infected cattle. If human bacilli will protect cattle, it is natural to suppose the converse-that bovine tubercle in children will protect them against human tubercle or phthisis.

I am at present engaged in collecting the blood of animals killed for tuberculosis, the serum of which, after careful preparation, I propose to use in vaccinating children whose parents have died of phthisis, with a view to protecting them against phthisis.

The brilliant results obtained by Professor Wright and other workers in the treatment of non-pulmonary forms of tubercle-such as glands, bones, genito-urinary, and lupus-with tuberculin may be explained, I venture to submit, by the fact that the tuberculin, being prepared from human bacilli, has an antagonistic effect on bovine forms of tubercle but has no effect on phthisis.

I conclude by expressing the opinion, based almost exclusively on clinical and pathological evidence, that human and bovine tuberculosis are distinct varieties of disease, but that the human body is susceptible to both, and especially to bovine tuberculosis in the milk-drinking period' of life.

\section{A CASE OF SUDDEN DEATH POSSIBLY DUE TO VAGUS INHIBITION.}

By E. D. TELFORD, M.A., B.C.Cantab., F.R.C.S.EnG., SURGEON, THE MANCHESTER HOSPITAL FOR SICK CHILDREN, PENDLEBURY.

THE features of the following case suggest that the sudden fatal ending may have been due to the damaged condition of the right vagus. A lesion in the position described would involve the thoracic cardiac branches of the right pneumogastric :

E. J., a pale, undersized girl of 11 years, was admitted to the Manchester Royal Infirmary on December 7th, 1903, suffering from chronic tuberculous cervical adenitis. The previous history of illness was negative, and there was no evidence of her ever having suffered from diphtheria. Glandular enlargeher ever having suffered from diphtheria. Glandular enlargement in the neck had existed for two years, and during the few weeks before admission the glands on the right side of the neck had discharged through two sinuses. The deep cervical
glands on both sides were enlarged, forming a diffuse indurated mass, reaching from the angle of the jaw to the inner end of the clavicle. The skin of the left side was intact, but on the right side it was much puckered and scarred. Below the angle of the jaw were two small sinuses with thin watery discharge. There was no other superficial glandular enlargement and no Ther of din sign of disease in the chest or abdomen. The pulse and temperature were normal. The source of the tuberculous in-
fection may have been the tonsils, which were slightly enlarged.

On December 11th the child was anaesthetized with chloroform, and the glands on the right side of the neck were widely removed through a free V-shaped incision. The operation proved difficult and tedious, and occupied one hour. A deep dissection was necessary and the large vessels were laid bare for some distance, but the right vagus nerve was not seen, The for some distance, but the right vagus nerve was not seen. The On two occasions the child's behaviour under the anaesthetic 